Acta Crystallographica Section E

Structure Reports

Online

ISSN 1600-5368

\section{5-Bromo-2-hydroxybenzaldehyde 4-ethylthiosemicarbazone}

\section{Zhaoyang Li and Osamu Sato*}

Institute for Materials Chemistry and Engineering, Kyushu University, 6-1 Kasugakoen, Kasuga, Fukuoka 816-8580, Japan

Correspondence e-mail: sato@cm.kyushu-u.ac.jp

Received 31 March 2013; accepted 1 April 2013

Key indicators: single-crystal X-ray study; $T=123 \mathrm{~K}$; mean $\sigma(\mathrm{C}-\mathrm{C})=0.005 \AA$;

$R$ factor $=0.042 ; w R$ factor $=0.114 ;$ data-to-parameter ratio $=15.0$.

In the title Schiff base compound, $\mathrm{C}_{10} \mathrm{H}_{12} \mathrm{BrN}_{3} \mathrm{OS}$, the $\mathrm{C}-\mathrm{N}-$ $\mathrm{N}-\mathrm{C}$ torsion angle is $172.07(11)^{\circ}$. An intramolecular hydrogen bond exists between the hydroxy $\mathrm{H}$ atom and the azomethine $\mathrm{N}$ atom. In the crystal, pairs of hydrogen bonds involving the imino $\mathrm{H}$ atom and the $\mathrm{S}$ atom give rise to supramolecular dimers.

\section{Related literature}

For the isostructural compound 5-chloro-2-hydroxybenzaldehyde 4-ethylthiosemicarbazone, see: Lo et al. (2011)<smiles>CCNC(=S)N/N=C/c1cc(Br)ccc1O</smiles>

\section{Experimental}

Crystal data

$\mathrm{C}_{10} \mathrm{H}_{12} \mathrm{BrN}_{3} \mathrm{OS}$

$M_{r}=302.20$

Monoclinic, $C 2 / c$

$$
\begin{aligned}
& a=22.040(4) \AA \\
& b=11.844(2) \AA \\
& c=9.5102(19) \AA
\end{aligned}
$$

$\beta=101.69(3)^{\circ}$

$V=2431.1(8) \AA^{3}$

$\mu=3.54 \mathrm{~mm}^{-1}$

$T=123 \mathrm{~K}$

$0.20 \times 0.10 \times 0.05 \mathrm{~mm}$

Mo $K \alpha$ radiation

\section{Data collection}

Rigaku Saturn70 diffractometer

Absorption correction: multi-scan (CrystalClear; Rigaku, 2008)

$T_{\min }=0.661, T_{\max }=0.838$

4201 measured reflections 2331 independent reflections 1760 reflections with $I>2 \sigma(I)$ $R_{\text {int }}=0.032$

Refinement

$R\left[F^{2}>2 \sigma\left(F^{2}\right)\right]=0.042$

$w R\left(F^{2}\right)=0.114$

$S=0.95$

2331 reflections

155 parameters

3 restraints

$\mathrm{H}$ atoms treated by a mixture of independent and constrained refinement

$\Delta \rho_{\max }=0.70 \mathrm{e}^{-3}$

$\Delta \rho_{\min }=-1.01{\mathrm{e} \AA^{-3}}^{-3}$

Table 1

Hydrogen-bond geometry $\left(\AA{ }^{\circ}\right)$.

\begin{tabular}{lllll}
\hline$D-\mathrm{H} \cdots A$ & $D-\mathrm{H}$ & $\mathrm{H} \cdots A$ & $D \cdots A$ & $D-\mathrm{H} \cdots A$ \\
\hline $\mathrm{O} 1-\mathrm{H} 1 A \cdots \mathrm{N} 1$ & $0.84(3)$ & $2.00(2)$ & $2.674(3)$ & $137(3)$ \\
$\mathrm{N} 2-\mathrm{H} 2 A \cdots \mathrm{S} 1^{\mathrm{i}}$ & $0.88(3)$ & $2.47(3)$ & $3.316(3)$ & $161(2)$ \\
$\mathrm{N} 3-\mathrm{H} 3 A \cdots \mathrm{S} 1^{\mathrm{ii}}$ & $0.87(3)$ & $2.75(3)$ & $3.510(3)$ & $146(3)$ \\
\hline
\end{tabular}

Symmetry codes: (i) $-x+\frac{3}{2},-y+\frac{3}{2},-z+1$; (ii) $x,-y+1, z+\frac{1}{2}$.

Data collection: CrystalClear (Rigaku, 2008); cell refinement: CrystalClear; data reduction: CrystalClear; program(s) used to solve structure: SHELXS97 (Sheldrick, 2008); program(s) used to refine structure: SHELXL97 (Sheldrick, 2008); molecular graphics: SHELXTL (Sheldrick, 2008); software used to prepare material for publication: publCIF (Westrip, 2010).

The authors would like to thank the China Scholarship Council (CSC).

Supplementary data and figures for this paper are available from the IUCr electronic archives (Reference: NG5322).

\title{
References
}

Lo, K. M. \& Ng, S. W. (2011). Acta Cryst. E67, o1453.

Rigaku (2008). CrystalClear. Rigaku Corporation, Tokyo, Japan.

Sheldrick, G. M. (2008). Acta Cryst. A64, 112-122.

Westrip, S. P. (2010). J. Appl. Cryst. 43, 920-925. 


\section{supporting information}

Acta Cryst. (2013). E69, o762 [https://doi.org/10.1107/S1600536813008787]

\section{5-Bromo-2-hydroxybenzaldehyde 4-ethylthiosemicarbazone}

\section{Zhaoyang Li and Osamu Sato}

\section{S1. Comment}

A Schiff ligand was synthesized through one-pot reaction with high yield using 5-bromo-2-hydroxybenzaldehyde and 4ethyl-3-thiosemicarbazide (Fig. 1). The title compound can be used as tridentate chelating ligand to construct spincrossover complexes. Isostructural 5-chloro-2-hydroxybenzaldehyde-4-ethylthiosemicarbazone was reported previously (Lo et al., 2011).

In the title compound, a strong intramolecular hydrogen bond $\mathrm{O}-\mathrm{H} \cdots \mathrm{N}$ is observed. An intermolecular $\mathrm{N}-\mathrm{H} \cdots \mathrm{S}$ hydrogen bond connects two molecules into a supramolecular dimer as shown in Figure 2.

\section{S2. Experimental}

5-Bromo-2-hydroxybenzaldehyde (4.02 g, $20 \mathrm{mmol})$ in $50 \mathrm{ml}$ ethanol and 4-ethyl-3-thiosemicarbazide (2.38 g, $20 \mathrm{mmol})$ were reacted for $6 \mathrm{~h}$ at $350 \mathrm{~K}$. Slow evaporation of the yellow solution gave large colorless crystals.

\section{S3. Refinement}

Carbon-bound $\mathrm{H}$-atoms were placed in calculated positions ( $\mathrm{C}-\mathrm{H} \mathrm{0.95,} 0.98$ and $0.99 \AA)$ and were included in the refinement in the riding model approximation, with $U_{\text {iso }}(\mathrm{H})=1.5 \mathrm{U}_{\mathrm{eq}}(\mathrm{C})$ for methyl $\mathrm{H}$ atoms and $1.2 \mathrm{U}_{\mathrm{eq}}(\mathrm{C})$ for the others. The hydroxy and amino $\mathrm{H}$ atoms were located in a difference Fourier map, and were refined with distance restraints of $\mathrm{O}$ $-\mathrm{H} 0.85 \pm 0.01$ and $\mathrm{N}-\mathrm{H} 0.88 \pm 0.01 \AA$; with $U_{\text {iso }}(\mathrm{H})=1.2 \mathrm{U}_{\mathrm{eq}}(\mathrm{N}$ and $\mathrm{O})$.

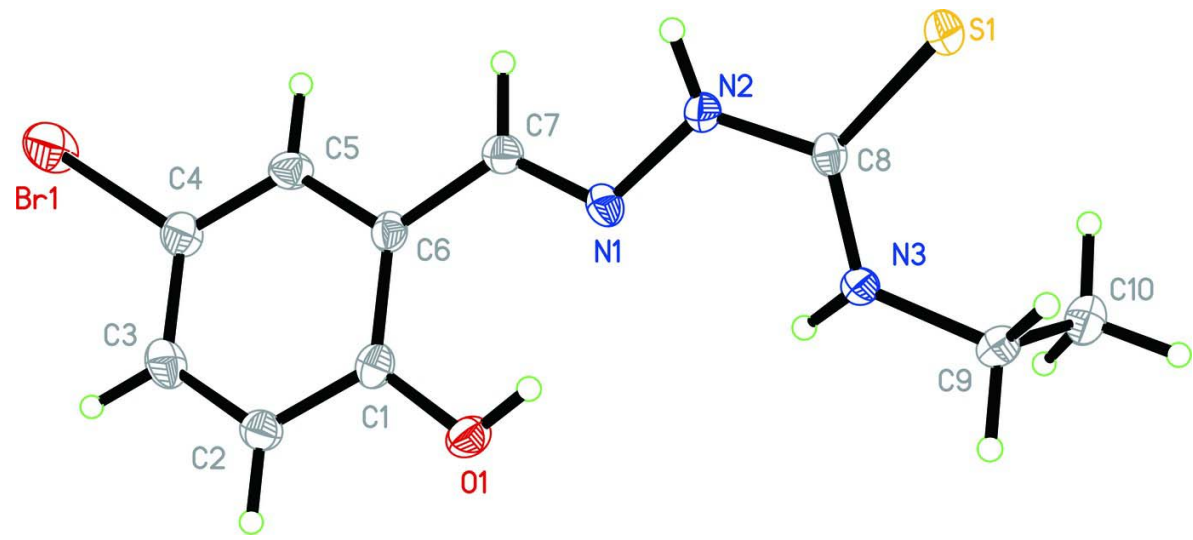

Figure 1

Displacement ellipsoid plot (50\% probability level) of the title compound, with atom numbering of structurally unique non- $\mathrm{H}$ atoms and the $\mathrm{H}$ atoms. 


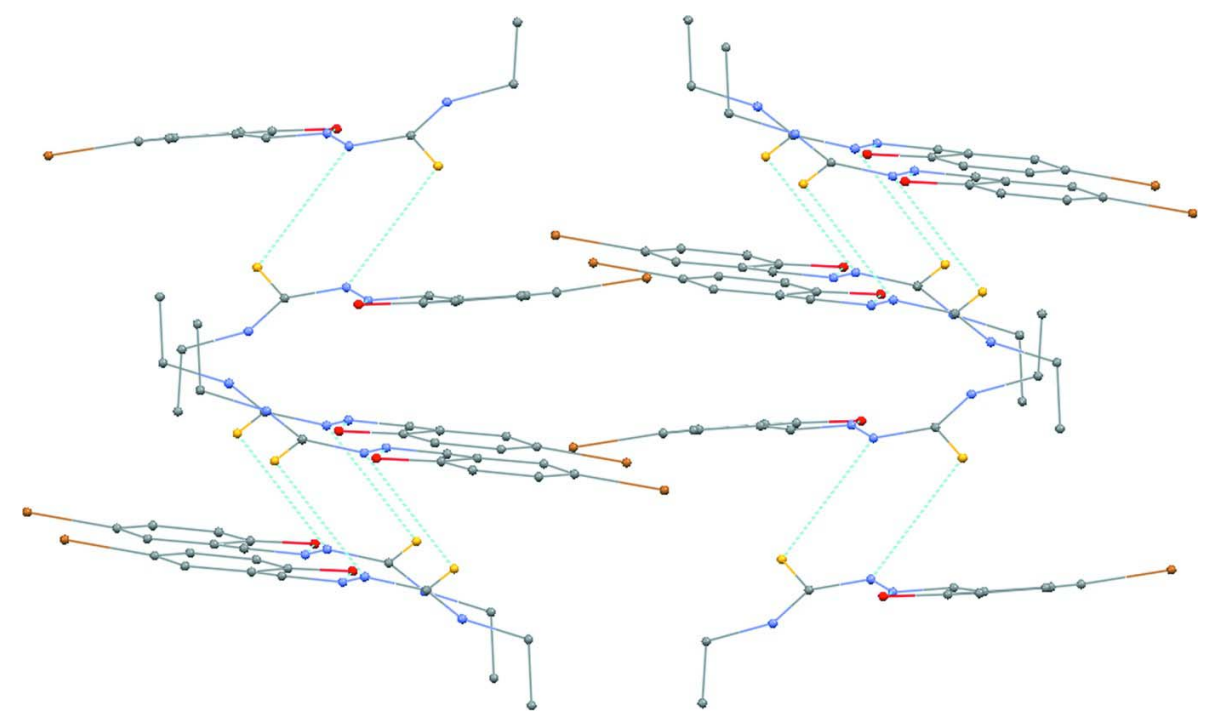

Figure 2

The packing diagram of the title compound, with $\mathrm{H}$ atoms omitted for clarity. Hydrogen bonds are shown as dashed lines.

5-Bromo-2-hydroxybenzaldehyde 4-ethylthiosemicarbazone

Crystal data

$\mathrm{C}_{10} \mathrm{H}_{12} \mathrm{BrN}_{3} \mathrm{OS}$

$M_{r}=302.20$

Monoclinic, $C 2 / c$

Hall symbol: $-\mathrm{C} 2 \mathrm{yc}$

$a=22.040$ (4) $\AA$

$b=11.844(2) \AA$

$c=9.5102(19) \AA$

$\beta=101.69(3)^{\circ}$

$V=2431.1(8) \AA^{3}$

$Z=8$

\section{Data collection}

Rigaku Saturn70 diffractometer

Radiation source: Rotating Anode

Confocal monochromator

Detector resolution: 28.5714 pixels $\mathrm{mm}^{-1}$

dtprofit.ref scans

Absorption correction: multi-scan

(CrystalClear; Rigaku, 2008)

$T_{\min }=0.661, T_{\max }=0.838$

\section{Refinement}

Refinement on $F^{2}$

Least-squares matrix: full

$R\left[F^{2}>2 \sigma\left(F^{2}\right)\right]=0.042$

$w R\left(F^{2}\right)=0.114$

$S=0.95$

2331 reflections

155 parameters

3 restraints
$F(000)=1216$

$D_{\mathrm{x}}=1.651 \mathrm{Mg} \mathrm{m}^{-3}$

Mo $K \alpha$ radiation, $\lambda=0.710747 \AA$

Cell parameters from 3650 reflections

$\theta=3.1-27.5^{\circ}$

$\mu=3.54 \mathrm{~mm}^{-1}$

$T=123 \mathrm{~K}$

Block, colourless

$0.20 \times 0.10 \times 0.05 \mathrm{~mm}$

4201 measured reflections

2331 independent reflections

1760 reflections with $I>2 \sigma(I)$

$R_{\text {int }}=0.032$

$\theta_{\max }=26.0^{\circ}, \theta_{\min }=3.1^{\circ}$

$h=-27 \rightarrow 20$

$k=-9 \rightarrow 14$

$l=-11 \rightarrow 10$

Primary atom site location: structure-invariant direct methods

Secondary atom site location: difference Fourier map

Hydrogen site location: inferred from neighbouring sites

$\mathrm{H}$ atoms treated by a mixture of independent and constrained refinement 
$w=1 /\left[\sigma^{2}\left(F_{\mathrm{o}}^{2}\right)+(0.0752 P)^{2}\right]$

where $P=\left(F_{\mathrm{o}}^{2}+2 F_{\mathrm{c}}^{2}\right) / 3$

$(\Delta / \sigma)_{\max }=0.001$

$$
\begin{aligned}
\Delta \rho_{\max } & =0.70 \mathrm{e} \AA^{-3} \\
\Delta \rho_{\min } & =-1.01 \mathrm{e} \AA^{-3}
\end{aligned}
$$

\section{Special details}

Geometry. All e.s.d.'s (except the e.s.d. in the dihedral angle between two 1.s. planes) are estimated using the full covariance matrix. The cell e.s.d.'s are taken into account individually in the estimation of e.s.d.'s in distances, angles and torsion angles; correlations between e.s.d.'s in cell parameters are only used when they are defined by crystal symmetry. An approximate (isotropic) treatment of cell e.s.d.'s is used for estimating e.s.d.'s involving 1.s. planes.

Refinement. Refinement of $F^{2}$ against ALL reflections. The weighted $R$-factor $w R$ and goodness of fit $S$ are based on $F^{2}$, conventional $R$-factors $R$ are based on $F$, with $F$ set to zero for negative $F^{2}$. The threshold expression of $F^{2}>\sigma\left(F^{2}\right)$ is used only for calculating $R$-factors(gt) etc. and is not relevant to the choice of reflections for refinement. $R$-factors based on $F^{2}$ are statistically about twice as large as those based on $F$, and $R$ - factors based on ALL data will be even larger.

Fractional atomic coordinates and isotropic or equivalent isotropic displacement parameters $\left(\hat{A}^{2}\right)$

\begin{tabular}{|c|c|c|c|c|}
\hline & $x$ & $y$ & $z$ & $U_{\text {iso }} * / U_{\text {eq }}$ \\
\hline Br1 & 0.484097 (17) & $0.67444(4)$ & $1.01744(4)$ & 0.04059 (19) \\
\hline $\mathrm{C} 1$ & $0.69767(15)$ & $0.6276(2)$ & $1.0597(3)$ & $0.0180(7)$ \\
\hline $\mathrm{C} 2$ & $0.67216(16)$ & $0.6364(3)$ & $1.1818(3)$ & $0.0197(7)$ \\
\hline $\mathrm{H} 2$ & 0.6985 & 0.6352 & 1.2742 & $0.024^{*}$ \\
\hline $\mathrm{C} 3$ & $0.60893(17)$ & $0.6470(3)$ & $1.1699(4)$ & $0.0227(7)$ \\
\hline $\mathrm{H} 3$ & 0.5919 & 0.6537 & 1.2536 & $0.027^{*}$ \\
\hline $\mathrm{C} 4$ & $0.57021(16)$ & $0.6476(3)$ & $1.0343(4)$ & $0.0222(7)$ \\
\hline $\mathrm{C} 5$ & $0.59471(16)$ & $0.6361(3)$ & $0.9125(3)$ & $0.0198(7)$ \\
\hline H5 & 0.5678 & 0.6343 & 0.8208 & $0.024^{*}$ \\
\hline C6 & $0.65841(15)$ & $0.6271(3)$ & $0.9228(3)$ & $0.0164(7)$ \\
\hline $\mathrm{C} 7$ & $0.68243(15)$ & $0.6269(3)$ & $0.7906(3)$ & $0.0179(7)$ \\
\hline $\mathrm{H} 7$ & 0.6542 & 0.6340 & 0.7012 & $0.021^{*}$ \\
\hline $\mathrm{C} 8$ & $0.81461(14)$ & $0.6124(2)$ & $0.6421(3)$ & $0.0150(6)$ \\
\hline C9 & $0.91521(15)$ & $0.5238(3)$ & $0.7381(3)$ & $0.0216(7)$ \\
\hline H9A & 0.9408 & 0.5182 & 0.8363 & $0.026^{*}$ \\
\hline H9B & 0.9339 & 0.5821 & 0.6855 & $0.026^{*}$ \\
\hline C10 & $0.91576(17)$ & $0.4111(3)$ & $0.6625(4)$ & $0.0270(8)$ \\
\hline H10A & 0.9008 & 0.3519 & 0.7190 & $0.040^{*}$ \\
\hline H10B & 0.9581 & 0.3935 & 0.6524 & $0.040^{*}$ \\
\hline $\mathrm{H} 10 \mathrm{C}$ & 0.8887 & 0.4152 & 0.5672 & $0.040^{*}$ \\
\hline $\mathrm{H} 1 \mathrm{~A}$ & $0.7730(17)$ & $0.634(3)$ & $1.002(2)$ & $0.032 *$ \\
\hline $\mathrm{H} 2 \mathrm{~A}$ & $0.7325(16)$ & $0.680(2)$ & $0.600(3)$ & $0.032^{*}$ \\
\hline $\mathrm{H} 3 \mathrm{~A}$ & $0.8346(17)$ & $0.525(3)$ & $0.810(3)$ & $0.032^{*}$ \\
\hline N1 & $0.74029(12)$ & $0.6174(2)$ & $0.7914(3)$ & $0.0169(6)$ \\
\hline $\mathrm{N} 2$ & $0.75628(13)$ & $0.6333(2)$ & $0.6594(3)$ & $0.0177(6)$ \\
\hline N3 & $0.85244(13)$ & $0.5580(2)$ & 0.7468 & $0.0172(6)$ \\
\hline $\mathrm{O} 1$ & 0.75977 (11) & 0.62315 (19) & $1.0781(2)$ & $0.0207(5)$ \\
\hline $\mathrm{S} 1$ & $0.83506(4)$ & $0.65794(7)$ & $0.48834(9)$ & $0.0201(2)$ \\
\hline
\end{tabular}


Atomic displacement parameters $\left(\AA^{2}\right)$

\begin{tabular}{lllllll}
\hline & $U^{11}$ & $U^{22}$ & $U^{33}$ & $U^{12}$ & $U^{13}$ & $U^{23}$ \\
\hline Br1 & $0.0165(2)$ & $0.0815(4)$ & $0.0261(2)$ & $0.00156(19)$ & $0.00976(16)$ & $0.00158(19)$ \\
C1 & $0.0186(18)$ & $0.0130(14)$ & $0.0228(17)$ & $0.0006(13)$ & $0.0052(14)$ & $-0.0009(13)$ \\
C2 & $0.0227(19)$ & $0.0187(15)$ & $0.0172(16)$ & $0.0008(13)$ & $0.0031(14)$ & $0.0002(13)$ \\
C3 & $0.026(2)$ & $0.0234(16)$ & $0.0223(16)$ & $-0.0019(14)$ & $0.0134(15)$ & $0.0023(14)$ \\
C4 & $0.0145(18)$ & $0.0314(18)$ & $0.0221(17)$ & $-0.0023(14)$ & $0.0066(14)$ & $-0.0001(14)$ \\
C5 & $0.0163(17)$ & $0.0239(16)$ & $0.0180(16)$ & $-0.0019(13)$ & $0.0010(13)$ & $0.0013(13)$ \\
C6 & $0.0173(17)$ & $0.0151(14)$ & $0.0180(16)$ & $0.0018(13)$ & $0.0065(13)$ & $0.0018(13)$ \\
C7 & $0.0176(17)$ & $0.0187(15)$ & $0.0172(15)$ & $0.0003(13)$ & $0.0032(13)$ & $0.0011(13)$ \\
C8 & $0.0166(17)$ & $0.0131(14)$ & $0.0162(15)$ & $0.0007(12)$ & $0.0056(13)$ & $-0.0023(13)$ \\
C9 & $0.0152(17)$ & $0.0291(17)$ & $0.0198(16)$ & $0.0029(14)$ & $0.0017(13)$ & $0.0026(14)$ \\
C10 & $0.021(2)$ & $0.033(2)$ & $0.0274(18)$ & $0.0060(15)$ & $0.0064(15)$ & $-0.0024(15)$ \\
N1 & $0.0195(15)$ & $0.0169(12)$ & $0.0158(13)$ & $-0.0005(11)$ & $0.0073(11)$ & $0.0006(11)$ \\
N2 & $0.0166(15)$ & $0.0213(13)$ & $0.0164(13)$ & $0.0046(11)$ & $0.0060(11)$ & $0.0034(11)$ \\
N3 & $0.0145(14)$ & $0.0227(14)$ & $0.0145(13)$ & $0.0024(11)$ & $0.0036(11)$ & $0.0030(11)$ \\
O1 & $0.0153(13)$ & $0.0259(12)$ & $0.0205(12)$ & $0.0017(10)$ & $0.0031(10)$ & $0.0040(10)$ \\
S1 & $0.0192(5)$ & $0.0253(4)$ & $0.0175(4)$ & $0.0038(3)$ & $0.0079(3)$ & $0.0032(3)$ \\
& & & & & &
\end{tabular}

Geometric parameters $\left(\AA,{ }^{\circ}\right)$

\begin{tabular}{|c|c|c|c|}
\hline $\mathrm{Br} 1-\mathrm{C} 4$ & $1.899(4)$ & $\mathrm{C} 8-\mathrm{N} 3$ & $1.329(4)$ \\
\hline $\mathrm{C} 1-\mathrm{O} 1$ & $1.345(4)$ & $\mathrm{C} 8-\mathrm{N} 2$ & $1.351(4)$ \\
\hline $\mathrm{C} 1-\mathrm{C} 2$ & $1.393(5)$ & $\mathrm{C} 8-\mathrm{S} 1$ & $1.703(3)$ \\
\hline $\mathrm{C} 1-\mathrm{C} 6$ & $1.410(5)$ & $\mathrm{C} 9-\mathrm{N} 3$ & $1.460(4)$ \\
\hline $\mathrm{C} 2-\mathrm{C} 3$ & $1.381(5)$ & $\mathrm{C} 9-\mathrm{C} 10$ & $1.517(5)$ \\
\hline $\mathrm{C} 2-\mathrm{H} 2$ & 0.9500 & $\mathrm{C} 9-\mathrm{H} 9 \mathrm{~A}$ & 0.9900 \\
\hline $\mathrm{C} 3-\mathrm{C} 4$ & $1.395(5)$ & C9-H9B & 0.9900 \\
\hline $\mathrm{C} 3-\mathrm{H} 3$ & 0.9500 & $\mathrm{C} 10-\mathrm{H} 10 \mathrm{~A}$ & 0.9800 \\
\hline $\mathrm{C} 4-\mathrm{C} 5$ & $1.380(5)$ & $\mathrm{C} 10-\mathrm{H} 10 \mathrm{~B}$ & 0.9800 \\
\hline $\mathrm{C} 5-\mathrm{C} 6$ & $1.391(4)$ & $\mathrm{C} 10-\mathrm{H} 10 \mathrm{C}$ & 0.9800 \\
\hline $\mathrm{C} 5-\mathrm{H} 5$ & 0.9500 & $\mathrm{~N} 1-\mathrm{N} 2$ & $1.384(3)$ \\
\hline $\mathrm{C} 6-\mathrm{C} 7$ & $1.460(4)$ & $\mathrm{N} 2-\mathrm{H} 2 \mathrm{~A}$ & $0.879(10)$ \\
\hline $\mathrm{C} 7-\mathrm{N} 1$ & $1.278(4)$ & $\mathrm{N} 3-\mathrm{H} 3 \mathrm{~A}$ & $0.876(10)$ \\
\hline $\mathrm{C} 7-\mathrm{H} 7$ & 0.9500 & $\mathrm{O} 1-\mathrm{H} 1 \mathrm{~A}$ & $0.846(10)$ \\
\hline $\mathrm{O} 1-\mathrm{C} 1-\mathrm{C} 2$ & $117.8(3)$ & $\mathrm{N} 3-\mathrm{C} 8-\mathrm{S} 1$ & $124.2(2)$ \\
\hline $\mathrm{O} 1-\mathrm{C} 1-\mathrm{C} 6$ & $122.5(3)$ & $\mathrm{N} 2-\mathrm{C} 8-\mathrm{S} 1$ & $118.0(2)$ \\
\hline $\mathrm{C} 2-\mathrm{C} 1-\mathrm{C} 6$ & $119.6(3)$ & $\mathrm{N} 3-\mathrm{C} 9-\mathrm{C} 10$ & $111.7(3)$ \\
\hline $\mathrm{C} 3-\mathrm{C} 2-\mathrm{C} 1$ & $120.6(3)$ & $\mathrm{N} 3-\mathrm{C} 9-\mathrm{H} 9 \mathrm{~A}$ & 109.3 \\
\hline $\mathrm{C} 3-\mathrm{C} 2-\mathrm{H} 2$ & 119.7 & $\mathrm{C} 10-\mathrm{C} 9-\mathrm{H} 9 \mathrm{~A}$ & 109.3 \\
\hline $\mathrm{C} 1-\mathrm{C} 2-\mathrm{H} 2$ & 119.7 & N3-C9-H9B & 109.3 \\
\hline $\mathrm{C} 2-\mathrm{C} 3-\mathrm{C} 4$ & $119.7(3)$ & $\mathrm{C} 10-\mathrm{C} 9-\mathrm{H} 9 \mathrm{~B}$ & 109.3 \\
\hline $\mathrm{C} 2-\mathrm{C} 3-\mathrm{H} 3$ & 120.2 & $\mathrm{H} 9 \mathrm{~A}-\mathrm{C} 9-\mathrm{H} 9 \mathrm{~B}$ & 107.9 \\
\hline $\mathrm{C} 4-\mathrm{C} 3-\mathrm{H} 3$ & 120.2 & $\mathrm{C} 9-\mathrm{C} 10-\mathrm{H} 10 \mathrm{~A}$ & 109.5 \\
\hline $\mathrm{C} 5-\mathrm{C} 4-\mathrm{C} 3$ & $120.4(3)$ & $\mathrm{C} 9-\mathrm{C} 10-\mathrm{H} 10 \mathrm{~B}$ & 109.5 \\
\hline $\mathrm{C} 5-\mathrm{C} 4-\mathrm{Br} 1$ & 120.0 & $\mathrm{H} 10 \mathrm{~A}-\mathrm{C} 10-\mathrm{H} 10 \mathrm{~B}$ & 109.5 \\
\hline
\end{tabular}




$\begin{array}{llll}\mathrm{C} 3-\mathrm{C} 4-\mathrm{Br} 1 & 119.5(3) & \mathrm{C} 9-\mathrm{C} 10-\mathrm{H} 10 \mathrm{C} & 109.5 \\ \mathrm{C} 4-\mathrm{C} 5-\mathrm{C} 6 & 120.6(3) & \mathrm{H} 10 \mathrm{~A}-\mathrm{C} 10-\mathrm{H} 10 \mathrm{C} & 109.5 \\ \mathrm{C} 4-\mathrm{C} 5-\mathrm{H} 5 & 119.7 & \mathrm{H} 10 \mathrm{~B}-\mathrm{C} 10-\mathrm{H} 10 \mathrm{C} & 109.5 \\ \mathrm{C} 6-\mathrm{C} 5-\mathrm{H} 5 & 119.7 & \mathrm{C} 7-\mathrm{N} 1-\mathrm{N} 2 & 114.8(3) \\ \mathrm{C} 5-\mathrm{C} 6-\mathrm{C} 1 & 119.1(3) & \mathrm{C} 8-\mathrm{N} 2-\mathrm{N} 1 & 120.6(3) \\ \mathrm{C} 5-\mathrm{C} 6-\mathrm{C} 7 & 118.4(3) & \mathrm{C} 8-\mathrm{N} 2-\mathrm{H} 2 \mathrm{~A} & 120(3) \\ \mathrm{C} 1-\mathrm{C} 6-\mathrm{C} 7 & 122.2(3) & \mathrm{N} 1-\mathrm{N} 2-\mathrm{H} 2 \mathrm{~A} & 116(3) \\ \mathrm{N} 1-\mathrm{C} 7-\mathrm{C} 6 & 122.0(3) & \mathrm{C} 8-\mathrm{N} 3-\mathrm{C} 9 & 123.4(3) \\ \mathrm{N} 1-\mathrm{C} 7-\mathrm{H} 7 & 119.0 & \mathrm{C} 8-\mathrm{N} 3-\mathrm{H} 3 \mathrm{~A} & 115(3) \\ \mathrm{C} 6-\mathrm{C} 7-\mathrm{H} 7 & 119.0 & \mathrm{C} 9-\mathrm{N} 3-\mathrm{H} 3 \mathrm{~A} & 119(3) \\ \mathrm{N} 3-\mathrm{C} 8-\mathrm{N} 2 & 117.8(3) & \mathrm{C} 1-\mathrm{O} 1-\mathrm{H} 1 \mathrm{~A} & 114(3)\end{array}$

Hydrogen-bond geometry $\left(A,{ }^{\circ}\right)$

\begin{tabular}{lllll}
\hline$D-\mathrm{H} \cdots A$ & $D-\mathrm{H}$ & $\mathrm{H} \cdots A$ & $D \cdots A$ & $D-\mathrm{H} \cdots A$ \\
\hline $\mathrm{O} 1-\mathrm{H} 1 A \cdots \mathrm{N} 1$ & $0.84(3)$ & $2.00(2)$ & $2.674(3)$ & $137(3)$ \\
$\mathrm{N} 2-\mathrm{H} 2 A \cdots \mathrm{S} 1^{\mathrm{i}}$ & $0.88(3)$ & $2.47(3)$ & $3.316(3)$ & $161(2)$ \\
$\mathrm{N} 3-\mathrm{H} 3 A \cdots \mathrm{S} 1^{\mathrm{ii}}$ & $0.87(3)$ & $2.75(3)$ & $3.510(3)$ & $146(3)$ \\
\hline
\end{tabular}

Symmetry codes: (i) $-x+3 / 2,-y+3 / 2,-z+1$; (ii) $x,-y+1, z+1 / 2$. 